\title{
The Role of Sleep Disruption and Negative Emotions as Risk Factors in Diabetes Management
}

\section{Gunjan Y. Trivedi ${ }^{1}$ Banshi Saboo ${ }^{2}$}

\author{
${ }^{1}$ Society for Energy and Emotions, Wellness Space, Ahmedabad, \\ Gujarat, India \\ ${ }^{2}$ Diacare, Ahmedabad, Gujarat, India.
}

J Soc Health Diab 2019;7:39-40

Risk factors for type 2 diabetes (T2D) are well documented: (1) physical inactivity, (2) family history, (3) unhealthy eating, and (4) overweight (obesity). ${ }^{1}$ For clinical management of T2D, the focus is on the modifiable factors to slow down the disease progression and manage the risk of complications (e.g., cardiovascular disease [CVD] or renal complications or neuropathy). In a way, beyond family history, all the modifiable factors are interrelated; that is, unhealthy eating and physical inactivity lead to obesity, which further affects disease progression. In the context of energy expenditure, this can be articulated as energy imbalance (between energy intake vs. energy expenditure) impacting the body weight (obesity) that increases the risk of T2D or creates challenges for management of existing T2D.

Based on research over the past few years, there is an opportunity to revisit the risk factors and its categories for T2D as the aforementioned factors are more physiological and new factors related to sleep imbalance and emotional imbalance (negative emotions) have emerged. These new risk factors contribute to homeostatic imbalance of the energy, nervous system, endocrine system and circulatory systems (including immune system). The findings below make a possible case for addition of (1) sleep disruption and (2) negative emotions (or depression) as additional risk factors for diabetes as well as its complications:

1. Poor-quality sleep affects the energy balance via modulation of neuroendocrine function and glucose metabolism. As a result, the body tends to increase the food intake and conserve the energy. In short, poor-quality sleep predisposes the risk of T2D., 2,3

2. Recent epidemiological and laboratory evidence confirms the previous findings of an association between sleep loss and increased risk of obesity. Studies have also demonstrated a correlation between self-reported sleep duration and increased incidence of chronic disease (incl. obesity, T2D, and CVD). 4.5

3. While the causal role of sleep disruption in T2D is not yet documented, there is sufficient evidence that type 2 diabetes mellitus (T2DM) and sleep disorders are common
Address for correspondence Gunjan Y. Trivedi, Wellness Space, 119C Swastik Society, Navrangpura, Ahmedabad 380 009, Gujarat, India (e-mail: gunjan@wellness-space.net).

problems that often coexist. The studies also have validated the need for T2DM patients to be screened for sleep disruption. ${ }^{6}$

4. Studies have also confirmed a reciprocal link between depression and obesity. Depression is an important risk factor not only in binge eating but also in development of obesity. At the same time, obesity is found to increase the depression risk. ${ }^{7}$ Further reviews also demonstrate a correlation between negative affect (mood) and increased glucose level. ${ }^{8-10}$

The emerging evidence of the links between sleep disruption, negative mood (or depression), and obesity provide new possibilities for social research in the area of chronic disease, especially T2D. For a healthy society and quality T2D management, following two areas could be added into future studies:

1. Sleep-related and daytime energy recovery, overall energy expenditure, and its correlation with obesity.

2. The role of emotional balance (specifically, reduction in negative affect) and its impact on obesity.

\section{Conflict of Interest}

None declared.

\section{References}

1 International Diabetes Federation Accessed March 6, 2019

2 Penev PD. Update on energy homeostasis and insufficient sleep. J Clin Endocrinol Metab 2012;97(6):1792-1801

3 Koren D, Dumin M, Gozal D. Role of sleep quality in the metabolic syndrome. Diabetes Metab Syndr Obes 2016;9:281-310

4 Beccuti G, Pannain S. Sleep and obesity. Curr Opin Clin Nutr Metab Care 2011;14(4):402-412

5 Nedeltcheva AV, Scheer FA. Metabolic effects of sleep disruption, links to obesity and diabetes. Curr Opin Endocrinol Diabetes Obes 2014;21(4):293-298

6 Khandelwal D, Dutta D, Chittawar S, Kalra S. Sleep disorders in type 2 diabetes. Indian J Endocrinol Metab 2017;21(5):758-761

7 Luppino FS, de Wit LM, Bouvy PF, et al. Overweight, obesity, and depression: a systematic review and meta-analysis of longitudinal studies. Arch Gen Psychiatry 2010;67(3):220-229

\section{received}

March 17, 2019

accepted

March 17, 2019
DOI https://doi.org/

10.1055/s-0039-1692372

ISSN 2321-0656.
C2019 Novo Nordisk Education Foundation
License terms

()(1) $\Theta \circledast$ 
8 Skaff MM, Mullan JT, Almeida DM, et al. Daily negative mood affects fasting glucose in type 2 diabetes. Health Psychol 2009;28(3):265-272

9 Tabák AG, Akbaraly TN, Batty GD, Kivimäki M. Depression and type 2 diabetes: a causal association? Lancet Diabetes Endocrinol 2014;2(3):236-245
10 Pan A, Keum N, Okereke OI, et al. Bidirectional association between depression and metabolic syndrome: a systematic review and meta-analysis of epidemiological studies. Diabetes Care 2012;35(5):1171-1180 